\section{Moving Forward}

Epigenetics involves changes in genome function that occur without a change in the DNA sequence. These changes include modifications to DNA such as cytosine methylation, histone modifications, and alterations in chromatin conformation that can change patterns of gene expression. Although the term epigenetics used to apply only to heritable patterns of gene regulation, it is now applied generally to these types of changes whether they have been proven to be heritable or not. According to John M. Greally, Associate Professor, Departments of Medicine and Molecular Genetics, Albert Einstein College of Medicine, Bronx, NY, the term epigenomics can be used to describe two areas of research: (i) the study of epigenetic regulation of gene expression using high-throughput techniques or (ii) the study of the influence of DNA sequence on epigenetic regulation. Human diseases in which epigenetic mechanisms are thought to play a part include aging, cancer, obesity, type 2 diabetes, and mental disorders.

Greally notes that researchers used to look at one active gene at a time. Methylation-sensitive restriction enzymes were used to detect methylated cytosine residues. Analysis of spots on two-dimensional (2-D) gels after electrophoresis of samples allows examination of a few thousand loci at best. Advances that have furthered research in this area include massively parallel, high-throughput sequencing and microarray technology. "This is where sequencing technology is going to be the biggest enabler," says Greally.

\section{NIH Roadmap}

The National Institutes of Health $(\mathrm{NIH})$, Bethesda, MD, recently announced that it will invest more than $\$ 190$ million over the next 5 years to accelerate epigenomic research as part of the $\mathrm{NIH}$

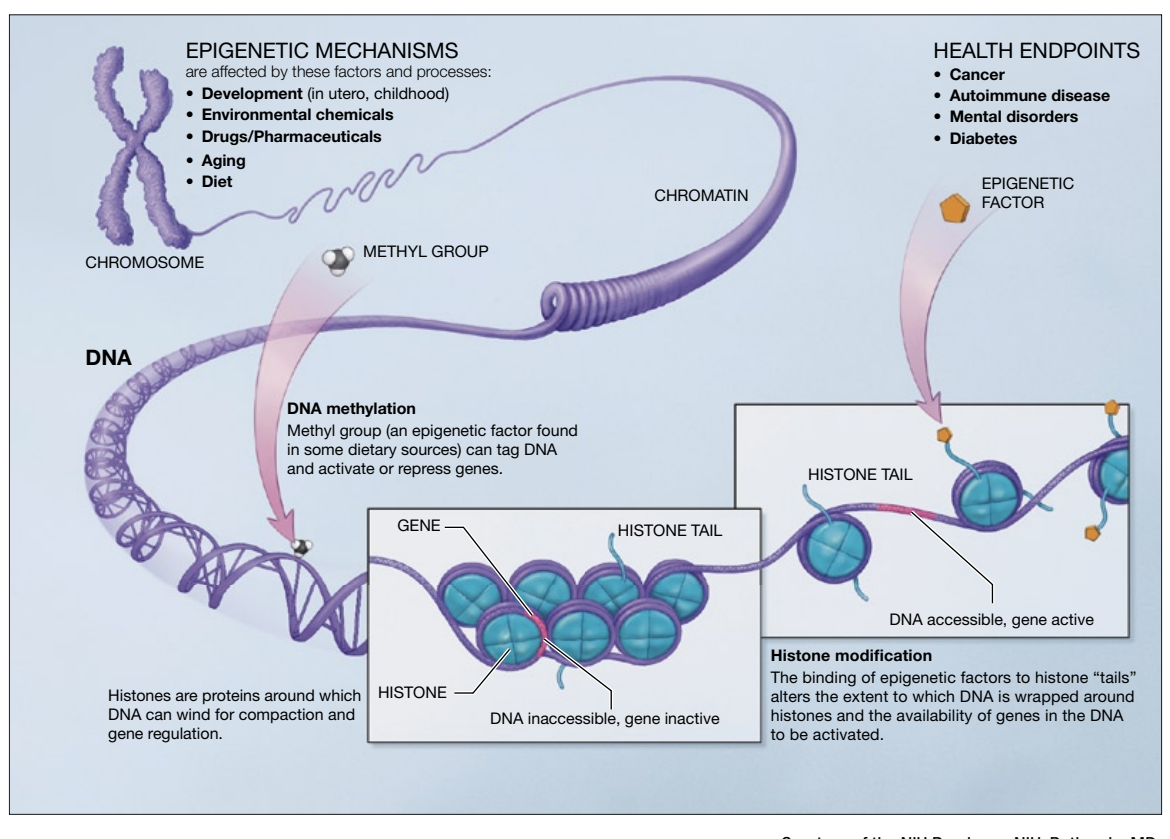

Courtesy of the NIH Roadmap, NIH, Bethesda, MD.

Scientific illustration of how epigenetic mechanisms can affect health.

Roadmap initiative. Funding is expected to promote research on epigenome mapping, data analysis, technology development, and discovery of novel mammalian epigenetic marks. The Epigenomics Program will function across several NIH institutes, including the National Institute of Environmental Health Sciences, the National Institute on Drug Abuse, the National Institute on Deafness and Other Communication Disorders, the National Institute of Diabetes and Digestive and Kidney Diseases, the National Institute of Neurological Disorders and Stroke, and the National Center for Biotechnology Information of the National Library of Medicine. This initiative is meant, in part, to expand research into epigenetic phenomenona beyond the current concentration on cancer into other diseases.

\section{Two Tracks and a Platform}

Greally divides epigenomic research into two tracks, one concerning what he calls the physiologic epigenome. Investigating the physiologic epigenome includes examination of his- tone modifications that vary among cell types, which can be enriched for using chromatin immunoprecipitation, and looking at DNA binding proteins. "I think this is a dominant way of gaining insights into the epigenome," says Greally. The other track concerns the epigenome as it is disregulated in human disease, particularly by methylation. He likens the use of histone immunoprecipitation to looking at the epigenome in color, and looking at cytosine methylation as viewing it in black and white. He thinks people had assumed that everything possible to know about cytosine methylation had been discovered, but it is being revisited. "We are obtaining interesting, counter-intuitive results," he says, "so we have a higher burden of proof to publish."

"What we're really doing is generating much more data than we can use," he observes. "It's not trivial, but it's easy to generate lots of data. We lack the ability to develop tools to visualize and analyze those data to develop biologic hypotheses." He views the epigenome 


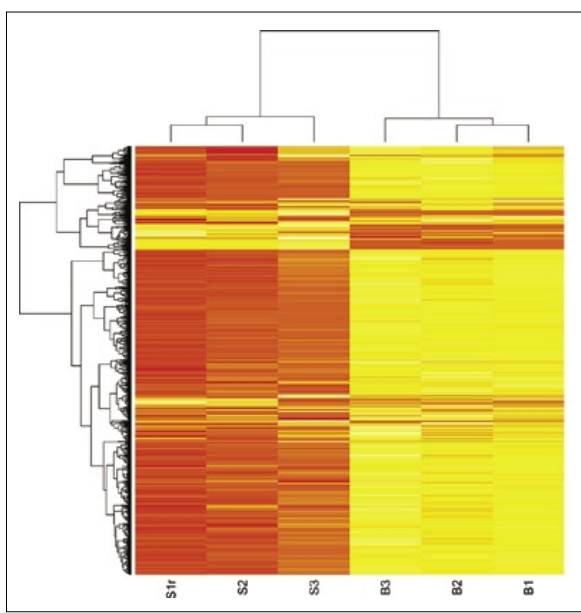

Data from Khulan et al. 2006. Genome Research 16(8):1046-55. Heatmap generated by Reid F. Thompson, Albert Einstein College of Medicine, Bronx, NY.

Unsupervised clustering of cytosine methylation profiles distinguishes the epigenomes of spermatogenic cells (S) from those of brain (B). Red, methylated; yellow, hypomethylated.

as a platform for systems biology in which mediators and interactions can be identified and defined, with new technology development to describe three-dimensional (3-D) interactions involving the genome. One thing that is particularly frustrating for people, Greally explains, is that unlike work with cell cultures, where one can have a gut sense of differences from one experiment to the next, feeding a sample into a high-throughput analyzer and receiving data doesn't necessarily give one a sense of what is going on. Junior researchers are starting to learn basic programming, he says, to make sense of what is going on at each stage. He would like to see companies provide raw data, which might be the basis of an open source movement in this field. He notes that most of the people in his laboratory spend more than half of their time analyzing data.

\section{Not All in the Mind}

Arturas Petronis, Associate Professor, Krembil Family Epigenetics Laboratory, Centre for Addiction and
Mental Health, Toronto, Canada, is interested in the epigenetics of complex non-Mendelian diseases. Although his laboratory focuses primarily on psychiatric disorders, they are also interested in other diseases. The role of epigenetics in these and other diseases is suggested by features that straightforward Mendelian inheritance cannot explain. These include discordance of phenotype between identical twins, as has been observed for mental disorders like schizophrenia. Epigenetic phenomena would be a good explanation for discordance between identical twins, environmental effects, differential susceptibility of males and females for disease (e.g., autoimmune disorders), parental origin effects of the same inherited gene, the course of some remitting and exacerbating diseases, and for disease where the mode of inheritance hasn't been elucidated.

\section{"What we're really doing is generating much more data than we can use."}

Petronis notes that although half the promoters may have $\mathrm{CpG}$ islands and are subject to regulation by methylation, there could be subtle differences between identical genomes that are not detectable by current microarray technology. He agrees that although methylation patterns are well-defined in cancers, other complex disorders have been "completely" ignored. In addition to looking at the epigenetics of major psychoses, his group is looking at type 1 diabetes, in which the genetic susceptibility or DNA sequence alone cannot explain development of the disease. He thinks that the location of the human insulin locus between genes subject to imprinting could be involved in development of autoimmunity to the insulin-producing $\beta$ cells of the pancreas. The group is also looking at involvement of the thymus in inactivation or not of anti- $\beta$ cell T cell clones in embryonic development.

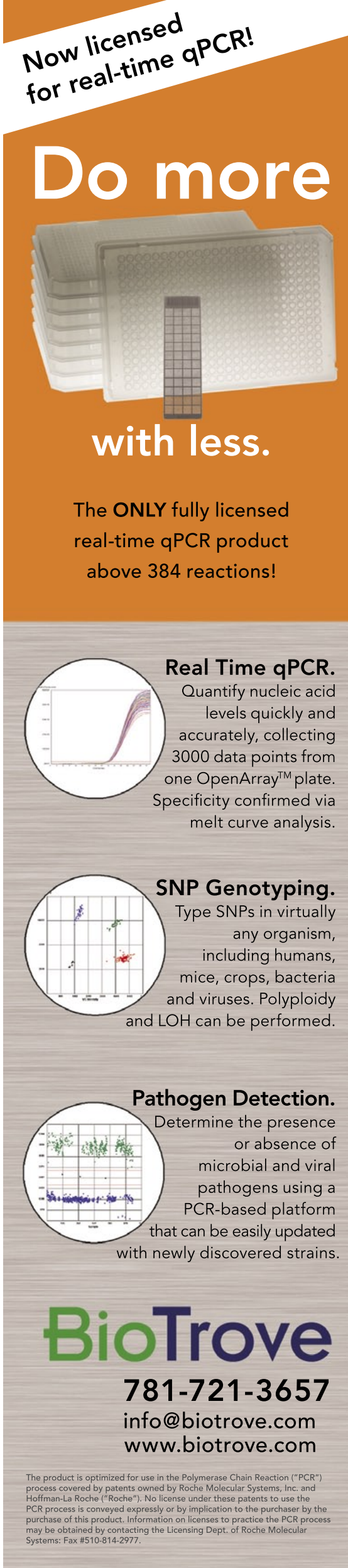

Circle Reader Service No. 133 
"Do we have all the technical tools we need? Yes and no," Petronis says. "We can perform concrete experiments. We have a superficial understanding of what genes to investigate, but we may be looking at where there's more light." The entire genome needs to be scanned, he says, and genome-wide linkage and association studies are needed. These are becoming more feasible, but the cost of the large number of microarrays required right now is "quite a bit of cash for an academic lab." Another bottleneck, he thinks, is that it is not clear what is the best way to enrich for methylated versus nonmethylated fractions of the genome.

\section{Current and Future Developments}

Peter A. Jones, Director, University of Southern California/Norris

Comprehensive Cancer Center, Los Angeles, CA, notes that in the last few months there has been interest in three "epigenetic drugs" currently approved. From a business standpoint, a major development, he says, is the size of buy-out offers for two companies that have developed demethylating agents used to treat patients with myelodysplastic syndrome (MDS). Another drug, vorinostat, a histone deacetylase inhibitor, was recently approved for treating cutaneous manifestations of $T$ cell lymphoma. A second huge development in the field of epigenomics is the interest in genomewide studies of histone modifications and of DNA methylation.

\section{"We're entering the golden age."}

Jones agrees with Greally that this "genomicizing" of epigenetics has been made possible by a big breakthrough in the advancement of chip and sequencing technology, particularly in the last 6 months. "This will change everything. The future,
I believe, is in an integrated approach," he says. Up until now, the various components of epigenetics, which he characterizes as a plastic entity, have been compartmentalized. He sees integrated epigenetic maps being developed, which will require a huge analytic capability. He believes that the NIH Roadmap Epigenomics initiative will push looking at histone modification, DNA methylation, and chromatin remodeling in its entirety.

Funding still isn't adequate, though, Jones thinks. He says that although it's been clear that the environment can influence the epigenome, "I certainly don't accept that change is transmissible through the germline." He thinks genetic transmission of epigenetic change is possible, but he remains skeptical and is not yet convinced. However, he acknowledges that epigenetics plays an important role in aging in general, as well as in late-onset diseases. "We're entering the golden age. The drugs are working. They are doubling the life span of patients with MDS."

It is not actually known why the demethylating drugs work, Jones concedes, because it's very hard to show that they actually induce changes in DNA methylation patterns. The patients don't care about the mechanism, he observes, if their lives are being extended. There is an ongoing push, of which he is a part, Jones concludes, to continue to develop new epigenetic-based therapies.

-Lynne Lederman is a freelance medical writer in Mamaroneck, NY.

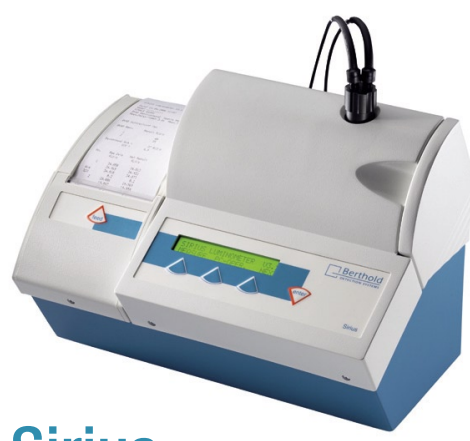

Sirius

Single Tube Luminometer 2 injectors, DLReady $^{\mathrm{TM}}$

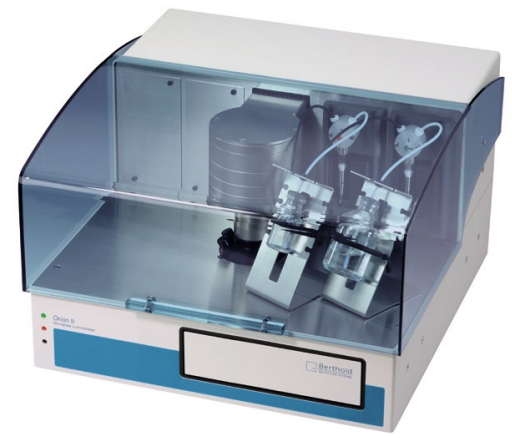

Orion II

Microplate Luminometer

4 injectors, $50^{\circ} \mathrm{C}$ incubation, 384 well, DLReady $^{\text {TM }}$
SENSITIVITY- Advanced photon counting for the best sensitivity.

FLEXIBILITY- Suitable for all known applications and easily adaptable to new requirements.

RELIABILITY - Quality workmanship, rigorous quality control, worldwide service and support.

VALUE - High quality instruments supplied in a plug-and play package, at a competitive price.

Berthold Detection Systems GmbH

Bleichstrasse 56-68, D-75173 Pforzheim

Phone: $+49(0) 7231 / 9206-0$

Berthold Detection Systems USA

144 Ridgeway Square, Oak Ridge, TN 37830

Phone: 888-522-5487 (USA)

www.berthold-ds.com

Circle Reader Service No. 135 